\title{
Isolated Acute Rotator Cuff Muscle Injury in Professional Baseball Players: 2 Case Reports
}

\section{Mamoru Yoshida ${ }^{1 *}$ and Hiroki Funasaki ${ }^{2}$}

${ }^{1}$ Associate Professor, Department of Orthopaedic Surgery, The Jikei University School

of Medicine, Minato-ku, Tokyo, Japan

${ }^{2}$ Professor, Department of Orthopaedic Surgery, The Jikei University School of

Medicine, Minato-ku, Tokyo, Japan

*Corresponding Author: Mamoru Yoshida, Associate Professor, Department of Orthopaedic Surgery, The Jikei University School of Medicine, Minato-ku, Tokyo, Japan.
Received: September 18, 2020

Published: September 29, 2020

(C) All rights are reserved by Mamoru

Yoshida and Hiroki Funasaki.

\section{Abstract}

Isolated acute injuries of scapulohumeral muscles are relatively rare in sports compared with general muscle injuries or compared with throwing-related disorders in shoulders. We had two retrospective cases of an isolated acute muscle injury to the subscapularis muscle developed in the pitching motion and to the infraspinatus muscle developed in the batting motion in professional baseball players during regular season games. Both cases were conservatively treated and successfully returned to play at their previous activity levels. We proposed that isolated acute injuries of rotator cuff muscles are relatively specific to professional baseball players, since there are few reports in non-professional players or in athletes in other kinds of sports.

Keywords: Rotator Cuff; Muscle Strain; Baseball; Pitching and Batting

\section{Introduction}

Isolated acute injuries of scapulohumeral muscles, which include subscapularis, supraspinatus, infraspinatus and teres minor, are relatively rare in sports compared with general muscle injuries in the lower extremities or trunk or compared with throwingrelated disorders of the shoulder, i.e. rotator cuff tendon tears, superior labrum anterior posterior (SLAP) tears or partial articular supraspinatus tendon avulsions (PASTA). In baseball players hamstring strains, rotator cuff injuries, SLAP tears or PASTA are often observed and those disorders were mainly caused with the imbalances between the amounts of mechanical stresses loaded in plays and player's physical conditions, i.e. restrictions of the range of motions in hips or shoulders, posterior tightness of shoulders or shoulder instabilities. Polster., et al. reported 10 cases of throwing-related injuries of the subscapularis muscle in professional baseball players, which included 7 pitchers and 3 outfielders, in a retrospective study [5]. We retrospectively reviewed two cases of isolated acute muscle injury in professional baseball players that occurred during games early in a regular season: one occurred in the subscapularis muscle during pitching and the other in the infraspinatus muscle during batting. Both cases were conservatively treated with therapy and successfully returned to play at their previous activity levels.

\section{Case Reports}

Informed consent was obtained from each patient for the review of their personal medical records, and institutional approval was obtained from the hospital's ethics committee (\#2020-12).

Case 1: An isolated acute injury of the subscapularis muscle during pitching

The patient was a 31-year-old male professional athlete playing as an overhand pitcher, right-handed, mainly pitching from the set position. When he pitched a fast ball in a game during the early reg- 
ular season from the set position, a moderate pain occurred in his right deep and central axilla during the phases between late cocking and early acceleration. He continued to pitch 7 more balls until the end of that game's inning with moderate axillar pain. He then left for our hospital because his axillar pain had become gradually more severe.

On initial physical examination there was tenderness in the central and deep axilla of his right shoulder but no active shoulder motions were disturbed in any direction. Pain was induced with passive abduction or external rotation of 90 degrees in his right shoulder and was also induced upon resistance against active internal rotation or active adduction. A lift off test, a bear hug test and a belly press test were negative. No obvious shoulder instability was observed in the anterior, inferior, or posterior directions. The range of motion (ROM) for horizontal flexion (HF) and internal rotation (IR) in a shoulder position of 90-degree abduction on his right, pitching side, shoulder were reduced approximately 15 degrees, compared with the same measurements of his left, nonpitching, shoulder. This indicated that there was slight posterior tightness in his right, pitching shoulder.

Plane X-P images showed no specific findings. Sagittal and coronal sections of T2-weighted magnetic resonance images (MRI) showed a heterogenous high intensity area at the myotendinous junction located in the inferior one-third of the subscapularis muscle in the axillar area. At the same time, there were no signs of injury or damage to the rotator cuff tendons, including subscapularis (Figure 1A and 1B). Axial sections of a T2-weighted MRI showed a slanted appearance, which was indicative of a bony defect on the posterior corner of the glenoid and a degradation of the posterior capsulolabral complex without any tendon injury to the subscapularis (Figure 1C).
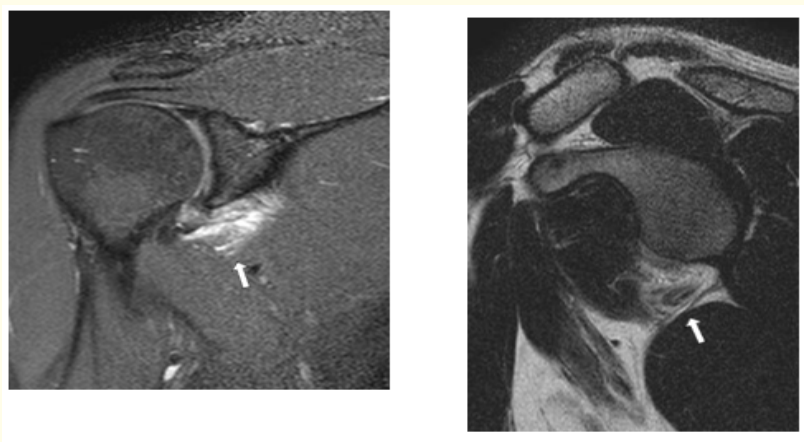

A

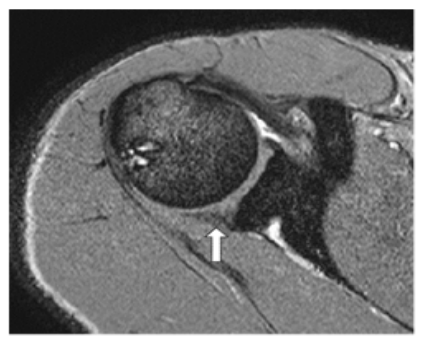

Figure 1: Magnetic resonance (MR) images of a right, pitching side shoulder in case 1. a) A coronal section with a T2-weighted MRI showing the heterogenous high intensity area located in the inferior one-third of the subscapularis muscle at the axillar area. b) A sagittal section with a T2-weighted MRI showing a heterogenous high intensity area located in the inferior one-third of the subscapularis muscle in the axillar area. c) A transverse section of a T2-weighted MRI showing a slant appearance at the posterior corner of the glenoid and no injury to the tendon of the subscapularis muscle.

From these findings the injury was diagnosed as an acute isolated injury of the inferior one-third of the subscapularis muscle with slight posterior tightness of his pitching shoulder. Conserva- tive therapy was chosen for the case. The patient was given a relative resting period of 5 days for his right shoulder and then he began practicing short-distance throwing and gradually increased in 
throwing distance. He simultaneously underwent a low intensity pulsed ultrasound (LIPUS) therapy in order to accelerate the healing of the damaged muscle and he was given mild shoulder stretching in all directions in order to treat a posterior contracture of his right shoulder. By 13 days after injury he had begun practicing his pitching and he returned to a professional baseball league game, pitching from the set position, at 20 days after injury, although slight discomfort remained in his axilla during pitching. No recurrence was observed through the end of the regular baseball league season.

Case 2: An isolated acute injury of the infraspinatus muscle during batting

The patient was a 33-year-old male professional baseball player who bats left and throws right as a field player. When he swung a bat with his maximum strength and missed hitting a fast ball pitched to the outside and high position in the strike zone during a game early in the regular season, a severe pain occurred in his right upper back. Since the severe pain continued and did not decrease, he left the game for our hospital.
On initial physical examination, no active shoulder motions were disturbed in any direction, and there was tenderness at the superior portion of the infraspinatus muscle on his right upper back. Pain was induced with passive internal rotation of his right shoulder or with passive horizontal flexion in a shoulder position of 90-degree abduction. Moreover, the pain was induced with resistance against active external rotation or active horizontal extension in a shoulder position of 90-degree abduction. An empty can test was positive, inducing pain in the upper portion of the infraspinatus muscle, and a full can test was negative. No shoulder instability was observed in any direction, and no laterality was observed in the ROM between shoulders.

Plane X-P images showed no specific findings. Magnetic resonance scans taken as T2-weighted images or taken in short tau inversion recovery (STIR) mode showed an abnormal linear high intensity area along the myotendinous junction, from distal to proximal, in the superior one-third of the infraspinatus muscle. Moreover, a paralabral cyst was observed behind the glenoid. Accompanying this, there were no signs of injury or damage to the rotator cuff tendons, including the infraspinatus in sagittal or axial sections (Figure 2).
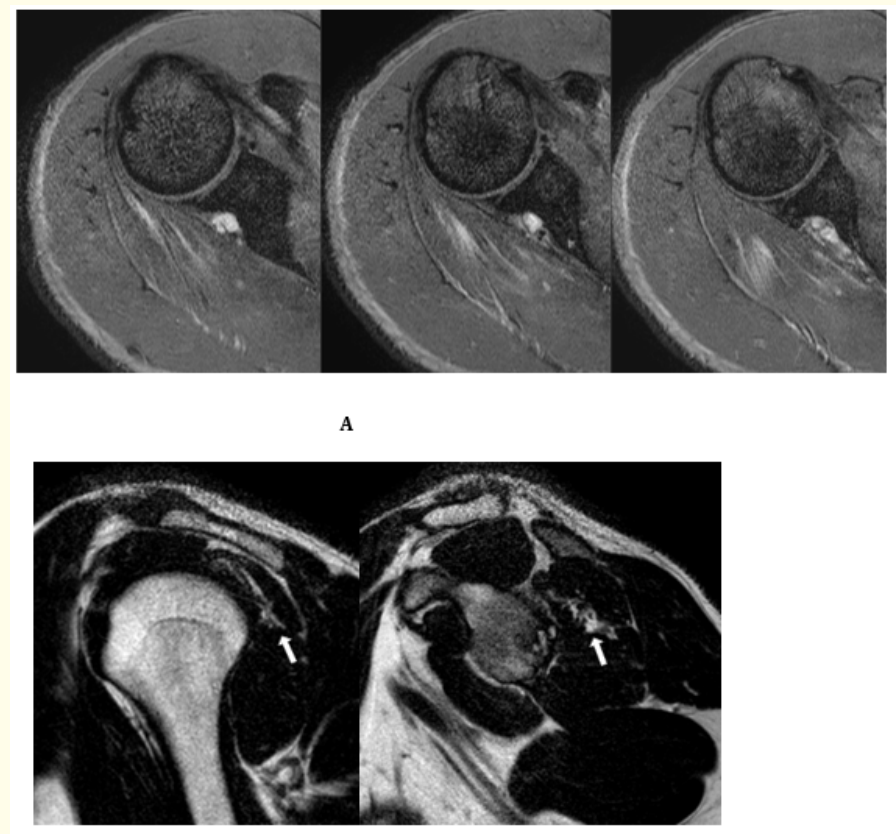

B

Figure 2: Magnetic resonance (MR) images of the right shoulder in case 2. a) Axial sections of a T2-weighted MRI showing several linear high intensity areas along the myotendinous junction from distal to proximal in the superior one-third portion of an infraspinatus muscle and a paralabral cyst located at the posterior aspect of the scapula behind glenoid without any injury or damage to the rotator cuff tendons. b) Sagittal sections of a T2-weighted MRI showing a high intensity area located along the myotendinous junction from distal to proximal in the superior one-third section of the infraspinatus muscle without any injury or damage to the rotator cuff tendons. 
From these findings the injury was diagnosed as an acute isolated injury of the infraspinatus muscle in the superior one-third of the myotendinous junction. It was diagnosed that a paralabral cyst did not correlated with the presence of symptoms since there was no symptoms before the present injury episode occurred. Conservative treatment with therapy was also chosen for this case. He underwent relative resting of his right shoulder for 5 days and then he began throwing short distances at 6 days after injury and gradually increased in throwing distance. He simultaneously underwent a LIPUS therapy in order to accelerate the healing of the damaged muscle and a mild shoulder stretching in all directions was simultaneously performed to prevent contracture of his right shoulder. He began practice swings at 7 days after injury and gradually increased in swing intensity with a bat. He started batting practice at 11 days after injury and returned to the game as a substitute batter or field player in the professional baseball league at 18 days after injury. No recurrence was observed through the end of the regular baseball league season.

\section{Discussion}

The incidence of isolated acute injury of the rotator cuff muscles is relatively low compared with that of general muscle injuries in sports. The proposed reason for this low incidence is that the main function of the scapulohumeral muscles is the maintenance of centralization of the humeral head during shoulder motion with several striated muscles, including the deltoid, pectoralis major and minor, teres major and latissimus dorsi, providing the main contributions toward active shoulder motions. In other words, the scapulohumeral muscles generally contribute less strength than the striated muscles do during active shoulder motions in sports. However, it is speculated that in professional baseball players, the contribution of the scapulohumeral muscles during shoulder motions is relatively greater than in non-professional players. This may be the reason throwing-related injuries of the subscapularis muscle are reported in professional baseball players, especially pitchers and outfielders [5] and not in non-professional baseball players or athletes in other kinds of sports. There is a possibility that isolated acute injuries of the scapulohumeral muscles are specific to professional baseball players, especially pitchers.

In case 1 it is postulated that the mechanism for the isolated injury of the subscapularis muscle has to do with the eccentric contraction and maximum tensile force load to the inferior portion of the muscle during maximal abduction and external rotation (ABER) between the late cocking and early acceleration phases of pitching $[1,2,5,8]$. In case 1 it is proposed that an excess of tension was loaded to the inferior portion of the subscapularis muscle compared to that which occurs during regular pitching, since he attempted to pitch the fastest ball he could at that time. Additionally, an MRI of his shoulder revealed a slant appearance, indicating the possibility of horizontal hyper-extension during pitching [6]. The location of the injured area in the subscapularis muscle, which was the inferior one-third section, was consistent with that found in 10 previously reported cases in the literature, where similar mechanisms of injury were proposed [5]. An isolated injury to the tendon of the subscapularis muscle is sometimes observed in sports, however, the reason the muscle and not the tendon was injured in case 1 remains uncertain.

To our knowledge, there are no reports of acute injuries to scapulohumeral muscles during batting in baseball. Electromyographic activity of the four rotator cuff muscles is relatively low compared to that of muscles in the hip or trunk, of which acute batting injuries are often observed [7]. One of the proposed mechanisms of injury in case 2 was a batter's shoulder. The definition of which includes a posterior labrum tear and posterior instability of the lead shoulder that occur after a bat swing misses hitting a pitch, especially an outside pitch $[3,4]$. In case 2 , this situation and the occurrence of injury was quite similar with that of a batter's shoulder. It has been proposed that the lack of counterforce that hitting the ball would provide, increases the force imparted on the posterior capsulolabral complex of the lead shoulder in a batter's shoulder $[3,4]$. However, in case 2 , there was no damage to the posterior capsulolabral complex and no posterior instability of his shoulder, which argues that the mechanism of injury in this case might be different than that of a batter's shoulder. The player in case 2 attempted to swing fast in order to hit a fast ball and he swung with a long radius to hit the ball pitched to an outside and high position. For this reason, the mechanism of injury in case 2 was speculated to include a greater tensile force load to the myotendinous junction of the infraspinatus muscle than the tension imparted during his regular swings, and that an eccentric contraction occurred. Another possible cause of injury may be that the contribution of this batter's infraspinatus muscle, in terms of strength imparted during batting, was relatively greater than that of other baseball batters.

Players in both case 1 and case 2 successfully returned to play at their previous activity level with conventional conservative therapy, without using any specific modalities. We suggest that the reason for this is that both muscle injuries were not severe, and both 
muscles were not main striated muscles, essential for best performance in playing baseball. Further study is necessary to clarify the precise injury mechanisms in detail in order to develop methods of preventative care.

\section{Conclusion}

Two rare cases of acute isolated rotator cuff strains were successfully treated with conservative therapies allowing the patients to return to previous sport activity levels of professional baseball players.

\section{Funding}

None.

\section{Declaration of Conflict of Interest}

I declare that I have no conflicts of interest in the authorship or publication of this contribution.

\section{Bibliography}

1. Ackland DC and Pandy MG. "Lines of action and stabilizing potential of the shoulder musculature". Journal of Anatomy 215 (2009): 184-197.

2. Kadaba MP., et al. "Intramuscular wire electromyography of the subscapularis". Journal of Orthopaedic Research 10 (1992): 394-397.

3. Kang RW., et al. "Posterior instability caused by batter's shoulder". Clinics in Sports Medicine 32.4 (2013): 797-802.

4. OKeefe KJ., et al. "Batter's shoulder: clinical outcomes and return to sport”. Cureus 12.4 (2020): e7681.

5. Polster JM., et al. "Throwing-related injuries of the subscapularis in professional baseball players". Skeletal Radiology 45.1 (2016): 41-47.

6. Sashi R. "Shoulder disorders in sports". In: Sashi R, Itoi E, Akita $K$, editors, MRI of the shoulder ( $2^{\text {nd }}$ edition) Tokyo, Medical view (2011): 173-174.

7. Shaffer B., et al. "Baseball batting. an electromyographic study". Clinical Orthopaedics and Related Research 292 (1993): 285-293.

8. Yanagisawa 0., et al. "Magnetic resonance imaging of the rotator cuff muscles after baseball pitching". The Journal of Sports Medicine and Physical Fitness 43 (2003): 493-499.

\section{Assets from publication with us}

- Prompt Acknowledgement after receiving the article

- Thorough Double blinded peer review

- Rapid Publication

- Issue of Publication Certificate

- High visibility of your Published work

Website: https://www.actascientific.com/

Submit Article: https://www.actascientific.com/submission.php Email us: editor@actascientific.com

Contact us: +919182824667 